RASĀYAN J. Chem.

Vol. 13 | No. 3 |1486-1490| July - September | 2020 ISSN: 0974-1496 | e-ISSN: 0976-0083 | CODEN: RJCABP

\title{
GAS SENSITIVITY SPEED OF AMMONIA SEMICONDUCTOR SENSORS BASED ON METAL-OXIDE FILMS $\mathrm{SiO}_{2} / \mathrm{TiO}_{2}-\mathrm{Fe}_{2} \mathrm{O}_{3}$
}

\author{
I.E. Abdurakhmanov ${ }^{1}$, O.A. Kuchkarov ${ }^{2, *}$ and E. Abdurakhmanov ${ }^{2}$ \\ ${ }^{1}$ Department of Inorganic Chemistry and Materials Science, Samarkand State University. \\ ${ }^{2}$ Department of Analytical Chemistry, Samarkand State University, Uzbekistan, 140104 \\ *E-mail: o-kuchkarov2018@mail.ru
}

\begin{abstract}
In the present article a sensor was investigated as a provider of the rapid determination of ammonia in a wide range of its concentrations in atmospheric air and process gases. It was found that the gas sensitivity value of the ammonia sensor depends on the amount of catalyst in the gas-sensitive material (GSM). An increase in the concentration of iron compounds in GSM from 5 to $10 \%$ leads to increase of the fast of sensors by 1.2-1.4 times. At an annealing temperature of $550^{\circ} \mathrm{C}$, the response time of the sensor reaches a stable value $(14 \mathrm{~s}$.) above which does not decrease the value of this indicator of the sensor.

Keywords: Ammonia, Sensor, Semiconductor, Catalyst, Titanium Oxide, Iron Oxide, Atmospheric Air, Gassensitive Material, Process Gases, Sensitivity, Sensor Response Time.
\end{abstract}

(C) RASĀYAN. All rights reserved

\section{INTRODUCTION}

The following advantages of semiconductor ammonia sensors: high sensitivity, fast, miniature, low cost make them very convenient for use as sensors for gas analytical devices. ${ }^{1}$ In this regard, the development of high efficient ammonia sensors in environmental sources is becoming an urgent task in chemistry and ecology.

\section{EXPERIMENTAL}

The principle of operation of a semiconductor ammonia sensor is based on a change in electrophysical properties of a sensitive layer consisting of titanium and iron oxides with the change in ammonia content in the analyzed gas medium. When oxygen is adsorbed on the surface of a semiconductor film, electrons pass from the semiconductor volume to the oxygen that is located on the surface, i.e. oxygen adsorbed on the surface of the grains causes the appearance of a depleted charge region and, at the same time, reduces or completely overlaps ping of the "isthmuses" between the grains, which are the channels for the movement of electrons. It should be noted that oxygen can be in various "charged" forms: $\mathrm{O}^{2}, \mathrm{O}^{-2}, \mathrm{O}^{-}$. For operating conditions, as a rule, a singly charged adsorption form of oxygen $\mathrm{O}^{-}$prevails. ${ }^{2}$ When ammonia interacts with negatively charged oxygen molecules on the surface of the semiconductor film electrons are formed, which transfer back into the semiconductor bulk, and the reaction products are removed from the surface in a neutral form. For example:

$$
2 \mathrm{NH}_{3}+3 \mathrm{O}^{-} \rightarrow 3 \mathrm{H}_{2} \mathrm{O}+\mathrm{N}_{2}+3 \mathrm{e} .
$$

Thus, the difference in the conductivities of the semiconductor layer in the absence and presence of ammonia in the surrounding atmosphere carries information about the concentration of the gas detected. In the work, for preparing low concentrations of ammonia in the air, a 667DG-03M diluent generator was used, which allows dilution of the source gas with air from 1/10 to $1 / 1000$. After mixing, the gas entered the chamber in which the studied sensor was installed. The gas flow fast was $50 \mathrm{ml} / \mathrm{min}$, and the pressure was close to atmospheric pressure. After gas passed through the chamber, traces of ammonia were removed from it in a trap with $20 \%$ sulfuric acid. 


\section{RESULTS AND DISCUSSION}

\section{Influence of the Temperature on the Sensitivity of a Semiconductor Ammonia Sensor}

For ammonia sensors based on semiconductor films, the optimal temperature for heating the GSM can vary. The optimum operating temperature ensures an acceptable signal value and the fast of the ammonia sensor. At high heating temperatures, large temperature gradients occur in the sensor, which leads to the failure of the sensor. In this regard, the development of low-temperature sensors is actual. For the same adsorbent, the sensitivity to various gases depends significantly on the temperature. This is because all reactions of sensitizing gas interaction (oxygen in a charged form) proceed with certain thermal effects. The electrical conductivity of the sensor changes due to the reactions of ammonia with chemisorbed oxygen in a charged form. There is a relatively narrow temperature range in which the adsorbent is sensitive to the gas which is detected. This may happen because at a low working temperature $t_{\text {work. }}$, the reaction products are not desorbed, i.e., the regeneration of oxygen adsorption centers is impossible. At high $t_{\text {work. }}$, adsorption of both oxygen and a reducing gas becomes impossible. In the work, the temperature dependence of the gas sensitivity of the $\mathrm{SiO}_{2} / \mathrm{TiO}_{2}$ и $\mathrm{SiO}_{2} / \mathrm{TiO}_{2}+\mathrm{Fe}_{2} \mathrm{O}_{3}$, where the $\mathrm{Fe}_{2} \mathrm{O}_{3}$ content is from 1 to $10 \%$, was studied for detecting ammonia. The influence of temperature on the gas sensitivity of the nanomaterial was studied in the temperature range from 50 to $500^{\circ} \mathrm{C}$. The results of establishing the effect of temperature on the sensitivity of the sensor are presented in Table-1.

Table-1: The Resistance Dependence of the Ammonia Sensor on the Temperature of the Heater

\begin{tabular}{c|c|c|c|c}
\hline \multirow{2}{*}{$\begin{array}{c}\text { The Temperature } \\
\text { of GSM, }{ }^{\circ} \mathrm{C}\end{array}$} & \multicolumn{4}{|c}{$\mathrm{GSM} \mathrm{composition}^{\left(\mathrm{C}_{\mathrm{NH}}=500 \mathrm{mg} / \mathrm{m}^{3}\right)}$} \\
\cline { 2 - 5 } & $\mathrm{SiO}_{2} / \mathrm{TiO}_{2-}$ & $\mathrm{SiO}_{2} / \mathrm{TiO}_{2}-1 \% \mathrm{Fe}_{2} \mathrm{O}_{3}$ & $\mathrm{SiO}_{2} / \mathrm{TiO}_{2}-5 \% \mathrm{Fe}_{2} \mathrm{O}_{3}$ & $\begin{array}{c}\mathrm{SiO}_{2} / \mathrm{TiO}_{2}-10 \% \\
\mathrm{Fe}_{2} \mathrm{O}_{3}\end{array}$ \\
\hline 50 & $\mathrm{R}_{\text {air }}=2760$ & $\mathrm{R}_{\text {air }}=2440$ & $\mathrm{R}_{\text {air }}=1830$ & $\mathrm{R}_{\text {air }}=1610$ \\
\hline 50 & 2713 & 2305 & 1604 & 1315 \\
\hline 100 & 2675 & 2283 & 1520 & 1202 \\
\hline 150 & 2635 & 2241 & 1413 & 893 \\
\hline 200 & 2610 & 2200 & 1290 & 753 \\
\hline 250 & 2585 & 2164 & 1125 & 680 \\
\hline 300 & 2537 & 2047 & 973 & 716 \\
\hline 350 & 2481 & 1995 & 933 & 795 \\
\hline 400 & 2455 & 1981 & 963 & 898 \\
\hline 450 & 2540 & 2056 & 1071 & 1207 \\
\hline 500 & 2590 & 2104 & & \\
\hline
\end{tabular}

The experimental data were used to calculate the change in the sensitivity of the layer (S) at different temperatures according to the equation: $\mathrm{S}=\left(\sigma_{\mathrm{gas}}-\sigma_{0}\right) / \sigma_{0}(1)$ where $\sigma$ gas is the electrical conductivity of the film when under the influence a gas of a given concentration; $\sigma_{0}$-electrical conductivity of the film in the air in the absence of gas. The results of calculations of the temperature dependence on the sensitivity of the gas-sensitive layer for ammonia are presented in Fig.-1.

As can be seen from Fig.-1, there was revealed the dependence of the sensitivity of ammonia semiconductor sensors on the temperature in non-monotonic and, in most cases, "bell-shaped" appearance. Based on the experiments results demonstrated in Fig.-1, the optimal value of the operating temperatures for each component of the GSM was determined, at which the sensor based on this active layer provides the highest signal values for ammonia. Thus, as a result of the experimental studies of the dependence of the conductivity of the developed sensors on the temperature, it has been found out that the highest signal on ammonia is observed in the presence of $\mathrm{SiO}_{2} / \mathrm{TiO}_{2}+10 \% \mathrm{Fe}_{2} \mathrm{O}_{3}$ at a temperature of $350^{\circ} \mathrm{C}$. It was also revealed that the "Thermal" constant of the sensor time, i.e. the output time of the resistance of the sensor sensitive layer in the temperature stabilization mode when the set of temperature changes from room temperature to $350^{\circ} \mathrm{C}$ for $3-5 \mathrm{~s}$. 
RASĀYAN J. Chem.

Vol. 13 | No. 3 |1486-1490| July - September | 2020

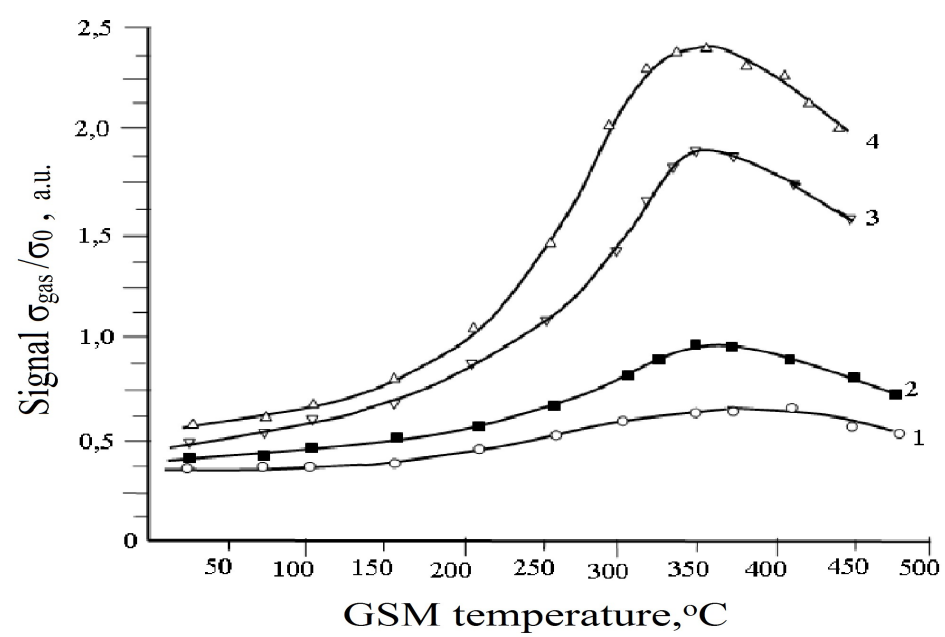

Fig.-1: Temperature Dependences of the Signal of a Gas-sensitive Material based on $\mathrm{SiO}_{2} / \mathrm{TiO}_{2}+1 \% \mathrm{Fe}_{2} \mathrm{O}_{3}$ Concerning Ammonia

$\left[1-\mathrm{SiO}_{2} / \mathrm{TiO}_{2} ; 2-\mathrm{SiO}_{2} / \mathrm{TiO}_{2}+1 \% \mathrm{Fe}_{2} \mathrm{O}_{3} ; 3-\mathrm{SiO}_{2} / \mathrm{TiO}_{2}+5 \% \mathrm{Fe}_{2} \mathrm{O}_{3} ; 4-\mathrm{SiO}_{2} / \mathrm{TiO}_{2}+10 \% \mathrm{Fe}_{2} \mathrm{O}_{3}\right]$

The Dependence of the Signal Value of the Ammonia Semiconductor Sensor on the Supply Voltage The value dependence of the $\mathrm{NH}_{3}$ signal on the sensor heater supply voltage has been studied in the range of 1.0-3.5 V. The experiments on the selection of optimal supply were carried out under ordinary conditions based on the example of a gas-air mixture with an ammonia concentration of $500 \mathrm{mg} / \mathrm{m}^{3}$. The obtained results for the selection of the optimal value of the ammonia sensor supply are presented in Table-2.

Table-2: The Dependence of the Signal $\left(\sigma_{\mathrm{gas}} / \sigma_{\text {air }}\right)$ of the Ammonia Sensor on the Heater Supply Voltage $\left(\mathrm{C}_{\mathrm{NH} 3}=500 \mathrm{mg} / \mathrm{m}^{3}\right)$

\begin{tabular}{|c|c|c|c|c|}
\hline \multirow{2}{*}{$\begin{array}{c}\text { The Sensor } \\
\text { Heater Supply, V }\end{array}$} & \multicolumn{4}{|c|}{ Composition GSM } \\
\hline & $\mathrm{SiO}_{2} / \mathrm{TiO}_{2-}$ & $\mathrm{SiO}_{2} / \mathrm{TiO}_{2}-1 \% \mathrm{Fe}_{2} \mathrm{O}_{3}$ & $\mathrm{SiO}_{2} / \mathrm{TiO}_{2}-5 \% \mathrm{Fe}_{2} \mathrm{O}_{3}$ & $\mathrm{SiO}_{2} / \mathrm{TiO}_{2}-1 \% \mathrm{Fe}_{2} \mathrm{O}_{3}$ \\
\hline \multicolumn{5}{|c|}{ The Sensor Signal $(\bar{x} \pm \Delta \mathrm{x})$} \\
\hline 1.2 & 1.03 & 1.07 & 1.2 & 1.34 \\
\hline 1.6 & 1.05 & 1.09 & 1.3 & 1.5 \\
\hline 1.8 & 1.06 & 1.11 & 1.42 & 1.8 \\
\hline 2.0 & 1.07 & 1.13 & 1.63 & 2.14 \\
\hline 2.3 & 1.09 & 1.19 & 1.88 & 2.37 \\
\hline 2.5 & 1.11 & 1.22 & 1.96 & 2.43 \\
\hline 2.8 & 1.12 & 1.23 & 1.90 & 2.25 \\
\hline 3.0 & 1.09 & 1.19 & 1.71 & 2.03 \\
\hline 3.3 & 1.07 & 1.16 & 1.52 & 1.79 \\
\hline
\end{tabular}

As it becomes vivid from the data of Table-2, the optimal power supply for the ammonia semiconductor sensor is $2.5 \mathrm{~V}$, which provides the temperature of $350^{\circ} \mathrm{C}$ on the sensor surface. The deviation of the supply value from the optimal value is accompanied by the decrease in the value of the useful analytical signal and the deterioration in selectivity.

The results of the study of the dependence of the signal $\left(\sigma_{\text {gas }} / \sigma_{\text {air }}\right)$ of the ammonia sensor on the supply voltage of the heater prove the possibility of ensuring the optimal value of the GSM surface temperature by changing the supply voltage of the sensor heater. It was found out that the optimal temperature of $350^{\circ} \mathrm{C}$ on the surface of the gas-sensitive material which is based on $\mathrm{SiO}_{2} / \mathrm{TiO}_{2}+10 \% \mathrm{Fe}_{2} \mathrm{O}_{3}$ corresponds to a heater supply voltage of $2.5 \mathrm{~V}$, where the maximum signal of the sensor is provided.

Dynamic Characteristics of the Semiconductor Ammonia Sensor

Considering the intended fields of applications and the high toxicity of ammonia, the main and satisfactory conditions for using the sensor should be created in a quick response time. It means that the 
RASĀYAN J. Chem.

Vol. 13 | No. 3 |1486-1490| July - September | 2020

ammonia sensor must have good dynamic characteristics. The value of the constant time of the sensors (fast), measured in the experiment, is determined by the processes in the adsorption layer, by the diffusion of gas molecules along with the thickness of the sensitive layer and the speed of replacement of the gas medium near the sensor. To increase the speed of the ammonia sensor, it is necessary to minimize the influence of the last two factors. In environmental monitoring and control of industrial emissions, the average concentrations of gaseous impurities are usually measured. For such measures, the sensor time constant of tens of seconds or several minutes is quite acceptable. ${ }^{3}$ The speed of the obtained thin-film samples concerning/the help of ammonia was measured using the equipment described above at temperatures of $200^{\circ} \mathrm{C}$. The experiments were carried out with an abrupt change in the concentration of ammonia at the input of the sensor. GS with ammonia concentrations of $50 \mathrm{mg} / \mathrm{m}^{3}$ was used in the experiments. The features of the ammonia sensor have been checked during continuous recording of the transient process of the chart tape of the KSP-4 recorder. The change in concentration at the input of SGS-NH $\mathrm{NH}_{3}$ was also noted on the diagram tape and was taken as the beginning of the time reference. The gas sensitivity value (S) was calculated by the formula (1). At the same time, the response time ( $\left.\mathrm{t}_{\mathrm{res}}\right)$ and the recovery time $\left(\mathrm{t}_{\mathrm{rec}}\right)$ of sensors based on GSM films of the composition $\mathrm{SiO}_{2} / \mathrm{TiO}_{2}+5 \% \mathrm{Fe}_{2} \mathrm{O}_{3}$ и $\mathrm{SiO}_{2} / \mathrm{TiO}_{2}+10 \% \mathrm{Fe}_{2} \mathrm{O}_{3}$ have been measured. The results of determining the dynamic features of SGS-NH are demonstrated in Fig.-2.

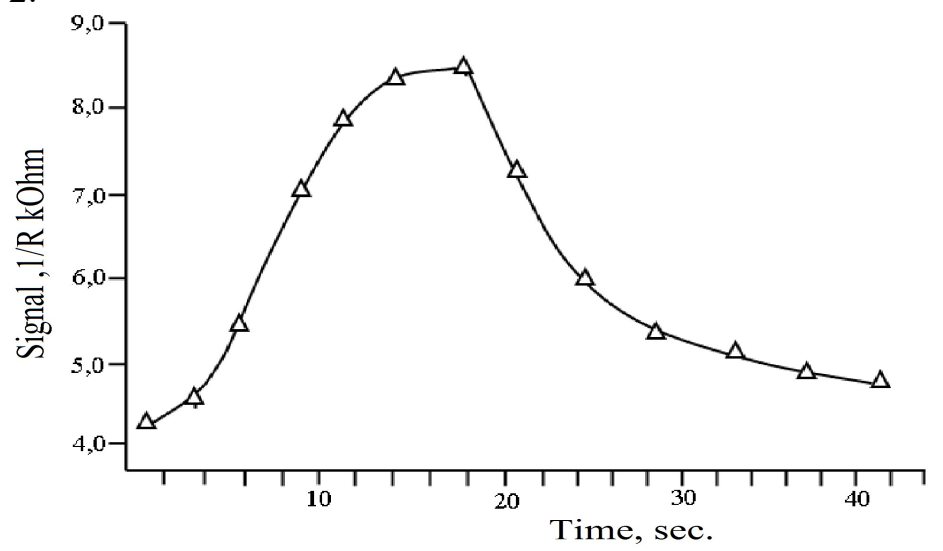

Fig.-2: The Response of the Sensor based on $\mathrm{SiO}_{2} / \mathrm{TiO}_{2}+10 \% \mathrm{Fe}_{2} \mathrm{O}_{3}$ Films Under the Influence of Ammonia $\left(\mathrm{T}_{\text {annel. }}=600{ }^{\circ} \mathrm{C}\right)$

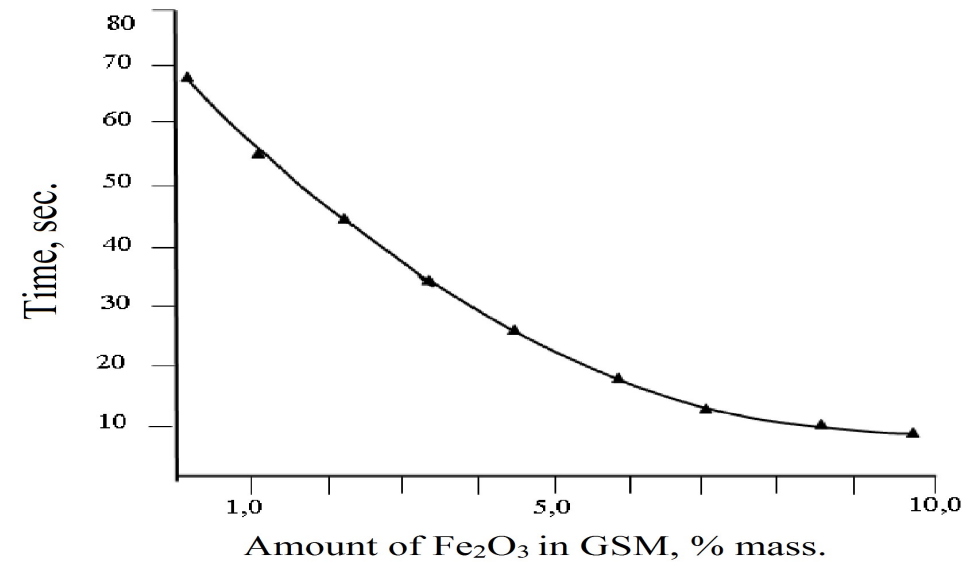

Fig.-3: Dependence of the Response (sec.) of the Ammonia Sensor on the Content of the Catalyst $\left(\mathrm{Fe}_{2} \mathrm{O}_{3}\right)$ in the GSM Annealed at the Temperature of $350^{\circ} \mathrm{C}$

Gas sensitivity studies have shown that a change in the surface resistance of the film in the direction of decrease is observed within 10-15 s after the ammonia enters the measuring chamber. Subsequent purging of the chamber with pure ammonia-free air returns the resistance to its original value. It was found that the gas sensitivity value, as well as $t_{\text {res }}$ and $t_{\text {ree }}$, depends on the content of the catalyst in GSM (Fig.-3). As 
the amount of iron oxide in the film increases, the response time decreases. The recovery time in purging the measuring chamber with air varies from 15 to $22 \mathrm{~s}$ and decreases with the increased concentration of iron oxide in the film. Therefore, the increase in the concentration of iron compounds from 5 to $10 \%$ leads to an increase in the fast gas sensors by 1.2-1.4 times.

The dependence of the response time of the semiconductor sensor based on $\mathrm{SiO}_{2} / \mathrm{TiO}_{2}+10 \% \mathrm{Fe}_{2} \mathrm{O}_{3}$ to 50 $\mathrm{mg} / \mathrm{m}^{3}$ of ammonia on the annealing temperature of the GSM is shown in Fig.-4.

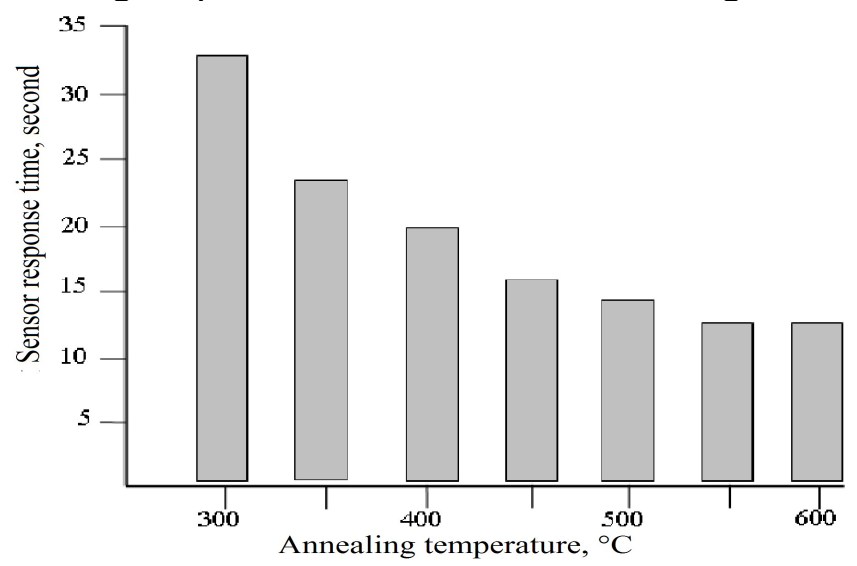

Fig.-4: Dependence of the Response Time of the Semiconductor Sensor based on $\mathrm{SiO}_{2} / \mathrm{TiO}_{2}+10 \% \mathrm{Fe}_{2} \mathrm{O}_{3}$ to 50 $\mathrm{mg} / \mathrm{m}^{3}$ of Ammonia on the Annealing Temperature of the GSM.

$\mathrm{SiO}_{2} / \mathrm{TiO} 2+10 \% \mathrm{Fe}_{2} \mathrm{O}_{3}$ films annealed at $300^{\circ} \mathrm{C}$ are characterized by insignificant gas sensitivity $(\mathrm{S}=0.06)$, in comparison with the samples annealed at $600^{\circ} \mathrm{C}(\mathrm{S}=0.17)$. The analysis of the response dependence (Fig. 4) of the film samples annealed at the temperature of $300-600^{\circ} \mathrm{C}$ showed that the sensor response time on them depends on the annealing temperature of the film and is reduced with increasing annealing temperature in the temperature interval from 300 to $550^{\circ} \mathrm{C}$. The minimum time constant $(14 \mathrm{~s})$ of the sensor based on $\mathrm{SiO}_{2} / \mathrm{TiO}_{2}+10 \% \mathrm{Fe}_{2} \mathrm{O}_{3}$ is reached at an annealing temperature of GSM of $550^{\circ} \mathrm{C}$. A further increase in the fast of the sensors turned out to be impossible because at higher temperatures the heater stability, the contacts to the sensitive layer and the heater are lost.

When recording the concentrations of ammonia in the air by selecting the amount of catalyst and increasing the annealing temperature of the GSM film to the maximum possible, it was possible to achieve a sensor performance based on titanium dioxide for about $14 \mathrm{~s}$.

\section{CONCLUSION}

Thus, the possibility of creating sensitive ammonia sensors using gas-sensitive materials based on titanium and iron oxides $\left(\mathrm{SiO}_{2} / \mathrm{TiO}_{2}+10 \% \mathrm{Fe}_{2} \mathrm{O}_{3}\right)$ has been experimentally confirmed. It was found that the gas sensitivity value, as well as $t_{\text {res }}$ and $t_{\text {rec }}$ of the ammonia sensor, depending on the amount of catalyst in GSM. An increase in the concentration of iron compounds in GSM from 5 to $10 \%$ leads to an increase in the fast of sensors by 1.2-1.4 times. At an annealing temperature of $550^{\circ} \mathrm{C}$, the response time of the sensor reaches a stable value (14s) above which does not lead to a decrease in the value of this indicator of the sensor.

\section{REFERENCES}

1. Technical Characteristics of the Sensors of Company Figaro Engineering, Technical Information for TGS 2442.

2. V. I. Gaman, 2012, Physics of Semiconductor Gas Sensors, Tomsk Publishing House of Scientific and Technical Literature, pp.110.

3. V. V. Petrov, Sensor, 147(2003).

[RJC-5718/2020] 